\title{
Clinical Guidelines for Managing Hearing Loss as a Complication of Drug-Resistant Tuberculosis Treatment: An Evaluation of Implementation fidelity in Kano, Nigeria
}

Sani Ibrahim Muhammad ( $\nabla$ mainagge2@gmail.com)

University of the Witwatersrand https://orcid.org/0000-0002-8849-2331

Ejemai Amaize Eboreime

University Of Alberta Department of Psychiatry https://orcid.org/0000-0001-8277-2570

Vivian Ifeoma Ogbonna

University of Port Harcourt Teaching Hospital

lliyasu Zubairu

Bayero University Faculty of Medicine: Bayero University College of Health Sciences

\section{Latifat Ibisomi}

University of the Witwatersrand School of Public Health

\section{Research}

Keywords: Adherence, Drug Resistance, Ototoxicity, Tuberculosis, Nigeria

Posted Date: July 8th, 2021

DOl: https://doi.org/10.21203/rs.3.rs-654798/v1

License: (c) (i) This work is licensed under a Creative Commons Attribution 4.0 International License.

Read Full License

Version of Record: A version of this preprint was published at BMC Health Services Research on February 3rd, 2022. See the published version at https://doi.org/10.1186/s12913-022-07536-y. 


\section{Abstract}

Background: Nigeria has a high burden of Tuberculosis (TB) including Drug-resistant Tuberculosis (DRTB) and hearing loss

Objective: To measure the implementation fidelity of management guidelines for hearing loss resulting from DR-TB treatment and to identify its determinants

Method: A questionnaire-based cross-sectional study was conducted at the Infectious Disease Hospital, Kano. Implementation fidelity of the Programmatic Management guidelines for the treatment of Drugresistant Tuberculosis was measured under the four domains of content, coverage, duration and frequency. The determinants examined are intervention complexity, facilitation strategies, quality of delivery and participant responsiveness as proposed by the Carroll et al framework. Other determinants used are age, sex, professional cadre and work experience of healthcare providers.

Results: The Implementation fidelity score ranged from $40-64 \%$ with a mean of $47.6 \%$. Quality of delivery, intervention complexity, participants' responsiveness, and being a medical doctor exerted a positive effect on implementation fidelity while facilitation strategy, age and work experience exerted a negative effect on implementation fidelity.

Conclusion: The implementation fidelity of management guidelines for hearing loss resulting from DR-TB treatment was low. Implementation fidelity should be assessed early and at intervals in the course of implementing the PMDT guideline and indeed, in the implementation of any intervention.

\section{Contributions To The Literature}

- Training and retraining, though important commonly employed implementation strategies, maybe counterproductive if used predominantly

- To improve fidelity in this context, efforts should focus on supportive supervision, coaching and mentorships.

- Such strategies may go a long way to reduce the incidence of hearing loss, thus improving patients compliance with the treatment regimen, and ultimately reducing the burden of drug resistant tuberculosis in Nigeria.

- This article contributes useful insights into application of implementation science approaches in the management of tuberculosis within the context of global health

\section{Introduction}

Hearing loss is a known complication of second-line drugs used in the management of Drug-Resistant Tuberculosis (DR-TB) (Jacobs \& Ross, 2012; World Health Organization, 2008). This complication is particularly common with injectable medications such as aminoglycosides, which often cause permanent 
damage to the hearing and balance systems of humans(Adeyemo et al., 2016; Sagwa et al., 2015). Yet these medications remain essential in the management of DR-TB, given the burden of the disease.

Nigeria has the tenth highest prevalence (323 per 100,000 people) of TB globally(World Health Organization, 2015, 2016). Despite several efforts directed toward its control, many patients fail to respond to treatment, having developed DR-TB(Enabudoso et al., 2006) - defined as resistance to at least one of the anti-TB drugs(Desai \& Joshi, 2017; World Health Organization, 2020). In 2017, the prevalence of DR-TB in Nigeria increased from $4.3-32 \%$ and from $25-53 \%$ for newly diagnosed and previously treated cases, respectively(Onyedum et al., 2017). This increase in prevalence raises concerns about meeting up with the target of providing access to DR-TB diagnosis to all presumptive DR-TB cases and enrolling $100 \%$ of diagnosed DR-TB patients on appropriate treatment, towards ending Tuberculosis by 2035(Onyedum et al., 2017; World Health Organization, 2011). Further concerns exist about the rise in the incidence of hearing loss resulting from intensified efforts to curb the rising cases of DR-TB. The incidence of hearing loss secondary to DR-TB management increased from $15.6 \%$ in 2004 to $61 \%$ in 2016 and may be contributing to poor patient compliance to the treatment regimen(Carroll et al., 2007).

The factors suggested for the rising incidence include (i) limited qualified personnel to implement the management guidelines, (ii) lack of appropriate audiological equipment for screening the patients on injectable second-line anti-TB drugs, (iii) health care personnel's negative attitude towards patients with hearing loss, and (iv) lack of adherence to the DR-TB guidelines/improper implementation of the guideline (Carroll et al., 2007).

The World Health Organization's (WHO) revised protocol for managing DR-TB called Programmatic Management of Drug-resistant Tuberculosis (PMDT) published in 2011 emphasizes monitoring and management of adverse effects resulting from the DR-TB treatment regimen(Onyedum et al., 2017). Nigeria adopted the guideline in 2012.(FMOH, 2010) The management of hearing is a component of the Nigerian TB Guideline (FMOH, 2008, 2010).

Whereas there are currently nine special centres established in Nigeria to carry out a hearing assessment in the treatment of DR-TB (Harris et al., 2012). Empirical evidence is lacking on how well the guidelines are being implemented nor on the factors affecting the implementation process. This study contributes to filling this gap by assessing the implementation fidelity of the management guidelines for hearing loss among healthcare providers at the DR-TB Centre of the Infectious Diseases Hospital, Kano State, Nigeria.

\section{Methods}

\section{Analytical Framework}

Implementation fidelity has been defined as "the degree to which programs are implemented as intended by the program developers"(Dusenbury et al., 2003). The conceptual framework in Figure 1 adapted from Carroll et al. guided the assessment of implementation fidelity and its determinants in this study(Carroll et al., 2007). Implementation fidelity has four constructs of duration, frequency, coverage and 
content(Ibrahim Muhammad, 2019). The four determinants affecting implementation fidelity according to the framework are: intervention complexity, facilitation strategies, quality of delivery and participant responsiveness(Barat et al., 2001).

Facilitation strategies refer to the delivery mechanism employed towards the effective implementation of an intervention. These strategies include training and the provision of protocols and guidelines. Quality of delivery typically describes the way the health care providers deliver the intervention. In this case, receiving supportive supervision, coaching and mentoring was used as a proxy measure of the quality of delivery of the PMDT management guideline. Intervention complexity describes the sophistication of an intervention, as simple, detailed intervention are very much likely to be delivered with high adherence (implementation fidelity) as compared to complex, vague interventions. Participants' responsiveness refers to the extent of participants' responses to the program or their level of engagement by it (here, PMDT management guideline). This often could be inferred from both the recipient of the intervention and the personnel responsible for delivering the intervention. Table 1 summarizes these analytical constructs. The following characteristics of the healthcare providers were also included in the model: age, sex, work experience and professional care.

\section{Study setting, design and population}

The study was conducted at the Infectious Disease Hospital (IDH), Kano, Nigeria. The IDH is a public specialized secondary health care facility serving a population of about 1.5 million people and having a patronage of about 300 patients/day. The hospital is the only infectious diseases hospital in the whole of Northern Nigeria and serves as a referral centre for Tuberculosis (TB) and handles the treatment of a patient with Drug-resistant Tuberculosis. Hearing loss assessment for DR-TB patients is conducted in IDH as part of PMDT guidelines' implementation. The implementation fidelity assessment was a crosssectional study. All front-line health care providers that have been involved in the implementation of PMDT guidelines for at least six months before the enquiry in the facility were recruited. Staff that met the inclusion criteria but were unavailable for whatever reason during the period of the study were excluded. A total of 73 health care providers were successfully interviewed.

This study was guided by the STrengthening the Reporting of OBservational studies in Epidemiology (STROBE) checklist (Additional file)

\section{Study questionnaire}

Data were collected through an interviewer-administered questionnaire. This was developed based on the domains and constructs of the adapted implementation fidelity framework. A 5-point Likert scale of "5Strongly agree, 4-agree, 3-neutral, 2-disagree and 1-strongly disagree" was used except for the facilitation strategy where Yes/No responses were used. The questionnaire was tested for reliability, as well as face and content validity in a pilot study. Implementation fidelity was measured by ten questions across its four constructs; facilitation strategy by six questions; quality of delivery by six questions; intervention 
complexity domain by three questions; and participants' responsiveness by four questions. Other information collected is age, sex, professional cadre and duration of working in PMDT (work experience).

\section{Data management and analysis}

The Cronbach-alpha reliability test was used to confirm the internal consistency of the questions used in measuring implementation fidelity and for each of the determinants. The alpha values ranged from 0.50 to 0.86 , indicating moderate to high internal consistency. This allows summation of the constructs to derive a composite score for each of the variables. The ten constructs of implementation fidelity had a minimum score of 10 and a maximum score of 50 . Likewise, scores were computed for each of the four core determinants. The values were then converted to percentages for analysis. The relationship between implementation fidelity and the determinants was examined using a linear regression model in Stata 14.2 (Inc. USA).

\section{Results}

Table 2 presents the basic characteristics of the respondents. Table 3 shows that the implementation fidelity score ranged from $40-64 \%$ with a mean of $47.6 \%$ and a median of $46 \%$. The range, mean with standard deviation and median with interquartile range for the four core determinates are also presented in Table 3. The unadjusted model in Table 4 shows that only quality of delivery, participants' responsiveness, sex and professional cadre were significantly associated with implementation fidelity.

In the adjusted model, facilitation strategies became significant and exerting a negative effect on implementation fidelity. Implementation fidelity decreases by 0.64 for every unit increase in facilitation strategy. On the other hand, for each unit increase in quality of delivery, implementation fidelity increased by 1.15 . Similarly, implementation fidelity increased by 1.24 with each unit increase in intervention complexity. Implementation fidelity score had a negative relationship with healthcare providers that were not medical doctors.

The eight predictors in the model explained about $77 \%$ of the variability in the model $\left(R^{2}=76.64\right)$. Thus, the characteristics measured in the model explained a good proportion of implementation fidelity in this context of management guidelines for hearing loss and its determinants.

\section{Discussion}

Using primary data, this study measured implementation fidelity of the management guideline for hearing loss resulting from the treatment of drug-resistant Tuberculosis among healthcare providers in the Infectious Disease Hospital, Kano, Nigeria. The study found the implementation fidelity to the PMDT guideline to be low among the 73 health care providers surveyed. The implementation fidelity was significantly influenced by facilitation strategies, quality of delivery, intervention complexity, and the professional cadre of the health care providers. Low implementation fidelity of the PMDT guideline among healthcare providers may account for the rise in the prevalence of hearing loss resulting from DR- 
TB treatment in Nigeria. The finding resonates with the concept that the degree to which the interventions are implemented affects the outcome of the intervention.(Dusenbury et al., 2003) Programs and interventions implemented with low fidelity usually lead to poor outcomes(Durlak \& DuPre, 2008; Proctor et al., 2011).

This study found that facilitation strategies (largely training of staff) hurt implementation fidelity. This finding is contrary to the expected positive influence that it should exert(Carroll et al., 2007; Roen et al., 2006). Thus, our study suggests that more training does not necessarily improve the implementation fidelity of interventions. From the literature, this observed negative effect may be attributable to training fatigue or the complexity of the training content or its delivery. Studies have established that programs are executed well with fidelity when they are simple, clear and detailed than when they are vague and nonspecific(Allen, 1998; Barat et al., 2001; Dusenbury et al., 2003). But further studies may be necessary to provide context-specific knowledge on the gaps concerning the PMDT implementation in Nigeria.

The availability of supportive supervision (quality of delivery) and clear roles in the management guidelines (intervention complexity) positively affected implementation fidelity in this study. Similarly, higher implementation fidelity of intervention is achieved when a program is acceptable to the participants responsible for the implementation and recipients of the intervention(Allen, 1998; Maidment et al., 2002). There is evidence from the literature that coaching, mentorship and supportive supervision is very effective in improving fidelity, acceptability and sustainability of the intervention(Eboreime \& BankeThomas, 2020; Sarre et al., 2018).

\section{Conclusion}

The study found implementation fidelity of management guideline for hearing loss resulting from Drugresistant Tuberculosis treatment to be low among health care providers at the infectious disease hospital, Kano, Nigeria. Training and retraining, though important, maybe counterproductive if used predominantly. To improve fidelity in this context, efforts should focus on supportive supervision, coaching and mentorships. Such strategies may go a long way to reduce the incidence of hearing loss, thus improving patients compliance with the treatment regimen, and ultimately reducing the burden of DR-TB in Nigeria.

\section{Declarations}

Conflict of Interest: None of the authors has any conflict of interest to declare.

\section{Ethics approval and consent to participate}

Ethical clearance was obtained from the Health Research Ethics Committee of the Kano State Ministry of Health (MOH/Off/797/T.I/620) and the Wits Human Research Ethics Committee (M171038), respectively. Informed consent was also obtained from each of the respondents.

\section{Consent for publication}


Not applicable

\section{Availability of data and materials}

The datasets used and/or analysed during the current study are available from the corresponding author on reasonable request.

\section{Competing interests}

None declared

\section{Funding}

This work has been funded by TDR, the Special Program for Research and Training in Tropical Diseases, which is hosted at the World Health Organization and co-sponsored by UNICEF, UNDP, the World Bank and WHO. TDR grant number: B40299.

\section{Authors' contributions}

SIM and LI conceptualized the study. SIM developed the study design. SIM did data collection, statistical analysis, and data interpretation with input from LI. SIM wrote the first draft of the manuscript. LI, EAE, VIO and ZI revised the manuscript critically for important intellectual content. All authors read and approved the final manuscript.

\section{Acknowledgements}

We acknowledge the management and staff of the Infectious Disease Hospital, Kano, Nigeria for theor support in this study.

\section{References}

1. Adeyemo AA, Oluwatosin 0, Omotade 00. Study of streptomycin-induced ototoxicity: protocol for a longitudinal study. SpringerPlus. 2016;5(1):1-9. https://doi.org/10.1186/s40064-016-2429-5.

2. Allen H. Promoting compliance with antihypertensive medication. British Journal of Nursing (Mark Allen Publishing). 1998;7(20):1252-8. https://doi.org/10.12968/bjon.1998.7.20.5563.

3. Barat I, Andreasen F, Damsgaard EMS. Drug therapy in the elderly: What doctors believe and patients actually do. Br J Clin Pharmacol. 2001;51(6):615-22. https://doi.org/10.1046/j.03065251.2001.01401.x.

4. Carroll C, Patterson M, Wood S, Booth A, Rick J, Balain S. A conceptual framework for implementation fidelity. Implementation Science. 2007;2(1):40. https://doi.org/10.1186/1748-59082-40.

5. Desai U, Joshi J. Unveiling the new definitions, diagnostic basis, and therapeutic approaches. Astrocyte. 2017;4(1):27. https://doi.org/10.4103/astrocyte.astrocyte_61_17. 
6. Durlak JA, DuPre EP. Implementation matters: A review of research on the influence of implementation on program outcomes and the factors affecting implementation. Am J Community Psychol. 2008;41(3-4):327-50. https://doi.org/10.1007/s10464-008-9165-0.

7. Dusenbury L, Brannigan R, Falco M, Hansen WB. A review of research on fidelity of implementation: implications for drug abuse prevention in school settings. Health Educ Res. 2003;18(2):237-56. https://doi.org/10.1093/her/18.2.237.

8. Eboreime EA, Banke-Thomas A. (2020). Beyond the Science: Advancing the "Art and Craft" of Implementation in the Training and Practice of Global Health. International Journal of Health Policy and Management, $x(\mathrm{x}), 1$-5. https://doi.org/10.34172/ijhpm.2020.131.

9. Enabudoso EJ, Gharoro EP, Ikena GO, Abhulimhen-lyoha B. Health and The Millennium Development Goals. The Nigerian Perspective. Benin Journal of Postgraduate Medicine. 2006;8(1):1-7. https://doi.org/10.1097/NMC.0000000000000166.

10. FMOH. (2008). Federal Ministry of Health The National Guidelines For TB Infection Control (pp. 133).

11. FMOH. (2010). Federal Ministry of Health of Nigeria - Department of Public Health. National Tuberculosis and Leprosy control Program (NTBLCP) Workers' Manual..

12. Harris T, Peer S, Fagan JJ. Audiological monitoring for ototoxic tuberculosis, human immunodeficiency virus and cancer therapies in a developing world setting. J Laryngol Otol. 2012;126(6):548-51. https://doi.org/10.1017/S0022215112000357.

13. Ibrahim Muhammad S. (2019). Implementation Fidelity of Management Guidelines for Hearing Loss Resulting From Treatment of Drug-Resistant Tuberculosis in Kano State, Nigeria (Issue July).

14. Jacobs TQ, Ross A. Adverse effects profile of multidrug-resistant tuberculosis treatment in a South African outpatient clinic. South African Family Practice. 2012;54(6):531-9. https://doi.org/10.1080/20786204.2012.10874288.

15. Maidment R, Livingston G, Katona C. Just keep taking the tablets": Adherence to antidepressant treatment in older people in primary care. Int J Geriatr Psychiatry. 2002;17(8):752-7. https://doi.org/10.1002/gps.688.

16. Onyedum CC, Alobu I, Ukwaja KN. Prevalence of drug-resistant tuberculosis in Nigeria: A systematic review and metaanalysis. PLoS ONE. 2017;12(7):1-17. https://doi.org/10.1371/journal.pone.0180996.

17. Proctor E, Silmere H, Raghavan R, Hovmand P, Aarons G, Bunger A, Griffey R, Hensley M. Outcomes for Implementation Research: Conceptual Distinctions, Measurement Challenges, and Research Agenda. Adm Policy Ment Health. 2011;38(2):65-76.

18. Roen K, Arai L, Roberts H, Popay J. Extending systematic reviews to include evidence on implementation: Methodological work on a review of community-based initiatives to prevent injuries. Soc Sci Med. 2006;63(4):1060-71. https://doi.org/10.1016/j.socscimed.2006.02.013.

19. Sagwa EL, Ruswa N, Mavhunga F, Rennie T, Leufkens HGM. Comparing amikacin and kanamycininduced hearing loss in multidrug-resistant tuberculosis treatment under programmatic conditions in 
a Namibian retrospective cohort. BMC Pharmacology Toxicology. 2015;16(36):1-9.

https://doi.org/10.1186/s40360-015-0036-7.

20. Sarre S, Maben J, Aldus C, Schneider J, Wharrad H, Nicholson C, Arthur A. The challenges of training, support and assessment of healthcare support workers: A qualitative study of experiences in three English acute hospitals. Int J Nurs Stud. 2018;79(November 2017):145-53. https://doi.org/10.1016/j.ijnurstu.2017.11.010.

21. World Health Organization. (2008). Guidelines for the programmatic management of drug-resistant tuberculosis Guidelines for the programmatic management of drug-resistant tuberculosis. World Health Organization, 12-185.

22. World Health Organization. (2011). GLOBAL TUBERCULOSIS CONTROL.

23. World Health Organization. (2015). Global Tuberculosis Report (Vol. 1). https://doi.org/10.1017/СВ09781107415324.004.

24. World Health Organization. (2016). Global tuberculosis report (Vol. 44, Issue 1).

25. World Health Organization. (2020). Definitions and reporting framework for tuberculosis - 2013 revision (updated Dec 2014 and Jan 2020) (WHO/ HTM/TB/2013.2). In Euro surveillance: bulletin Européen sur les maladies transmissibles = European communicable disease bulletin (Issue 16).

\section{Tables}

Table 1: Analytical constructs and their operational definitions in this study

\begin{tabular}{|c|c|}
\hline Analytical construct & Operational definition in this study \\
\hline Implementation fidelity & $\begin{array}{l}\text { Adherence to the Programmatic Management of Drug-resistant } \\
\text { Tuberculosis guidelines }\end{array}$ \\
\hline Facilitation strategies & $\begin{array}{l}\text { Training on and provision of protocols and guidelines to health care } \\
\text { providers }\end{array}$ \\
\hline Quality of delivery & $\begin{array}{l}\text { Supportive supervision, coaching and mentorship for implementation of } \\
\text { the guidelines }\end{array}$ \\
\hline Intervention complexity & $\begin{array}{l}\text { Perceived difficulty of implementing the guidelines by health care } \\
\text { providers }\end{array}$ \\
\hline $\begin{array}{l}\text { Participants' } \\
\text { responsiveness }\end{array}$ & $\begin{array}{l}\text { The extent to which the health care providers understand the content of } \\
\text { the guidelines }\end{array}$ \\
\hline
\end{tabular}

Table 2: Basic characteristics of study population 


\begin{tabular}{|ll|}
\hline Variable & N (\%) \\
\hline Age category & \\
\hline$\leq 35$ & $28(38.4)$ \\
$>35$ & $45(61.6)$ \\
\hline Sex & \\
\hline Male & $14(19.2)$ \\
\hline Female & $59(80.8)$ \\
\hline Professional cadre & \\
\hline Doctors & $13(17.8)$ \\
\hline Nurses & $49(67.1)$ \\
\hline Principal Clinical Assistant & $11(15.1)$ \\
\hline Work experience & \\
\hline$<12$ months & $10(13.7)$ \\
\hline 13 - 36 months & $17(23.3)$ \\
\hline$>36$ months & $46(63.0)$ \\
\hline
\end{tabular}

Table 3: Implementation fidelity scores

\begin{tabular}{|llll|}
\hline Va1iables & $\begin{array}{l}\text { Range } \\
\%\end{array}$ & Mean (SD) & Median (IQR) \\
\hline Implementation fidelity & $40-64$ & $47.6(5.7)$ & $46(42-52)$ \\
\hline Facilitation strategy & $17-100$ & $78(27.1)$ & $83(83-100)$ \\
\hline Quality of delivery & $06-20$ & $15(04.3)$ & $17(13-19)$ \\
\hline Intervention complexity & $20-60$ & $41(13.8)$ & $47(27-53)$ \\
\hline Participants' responsiveness & $25-45$ & $36(06.8)$ & $40(30-40)$ \\
\hline
\end{tabular}

SD: Standard deviation

IQR: Inter quartile range 
Table 4: Regression outputs of implementation fidelity and its determinants

\begin{tabular}{|c|c|c|c|c|}
\hline \multirow[t]{2}{*}{ Factors } & \multicolumn{2}{|c|}{ Unadjusted Model } & \multicolumn{2}{|c|}{ Adjusted $\left(R^{2}=76.64\right)$} \\
\hline & Coefficient & $95 \% \mathrm{Cl}$ & Coefficient & $95 \% \mathrm{Cl}$ \\
\hline Facilitation strategies & 0.18 & $-0.31-0.68$ & $-0.64^{\star}$ & $-1.23-0.05$ \\
\hline Quality of delivery & $1.91 *$ & $1.11-2.71$ & $1.15^{\star}$ & $0.17-2.12$ \\
\hline Intervention complexity & -0.69 & $-1.65-0.27$ & $1.24^{\star}$ & $0.53-1.95$ \\
\hline Participants' responsiveness & $3.82^{\star}$ & $2.07-5.56$ & 0.57 & $-1.88-3.01$ \\
\hline Age & 0.32 & $-1.76-2.41$ & -1.13 & -1.13 \\
\hline Work experience & 0.45 & $-0.29-1.21$ & -0.14 & -0.14 \\
\hline \multicolumn{5}{|l|}{ Sex } \\
\hline Female (Ref) & 0.00 & 0.00 & & \\
\hline Male & $10.37^{\star}$ & $8.03-12.71$ & 0.67 & 0.67 \\
\hline \multicolumn{5}{|l|}{ Professional Cadre } \\
\hline Doctors (Ref) & 0.00 & & 0.00 & \\
\hline Nurses & $-11.31^{\star}$ & $-13.67--8.95$ & $-12.80 *$ & $-12.80 *$ \\
\hline PCA & $-9.34^{\star}$ & $-12.45--6.24$ & $-13.25^{\star}$ & $-13.25^{\star}$ \\
\hline
\end{tabular}

* = Statistical significance at $5 \%$ level.

$\mathrm{R}^{2}=$ the value accounting for the internal variation of implementation fidelity in the model

\section{Figures}




\section{Framework guiding the study}

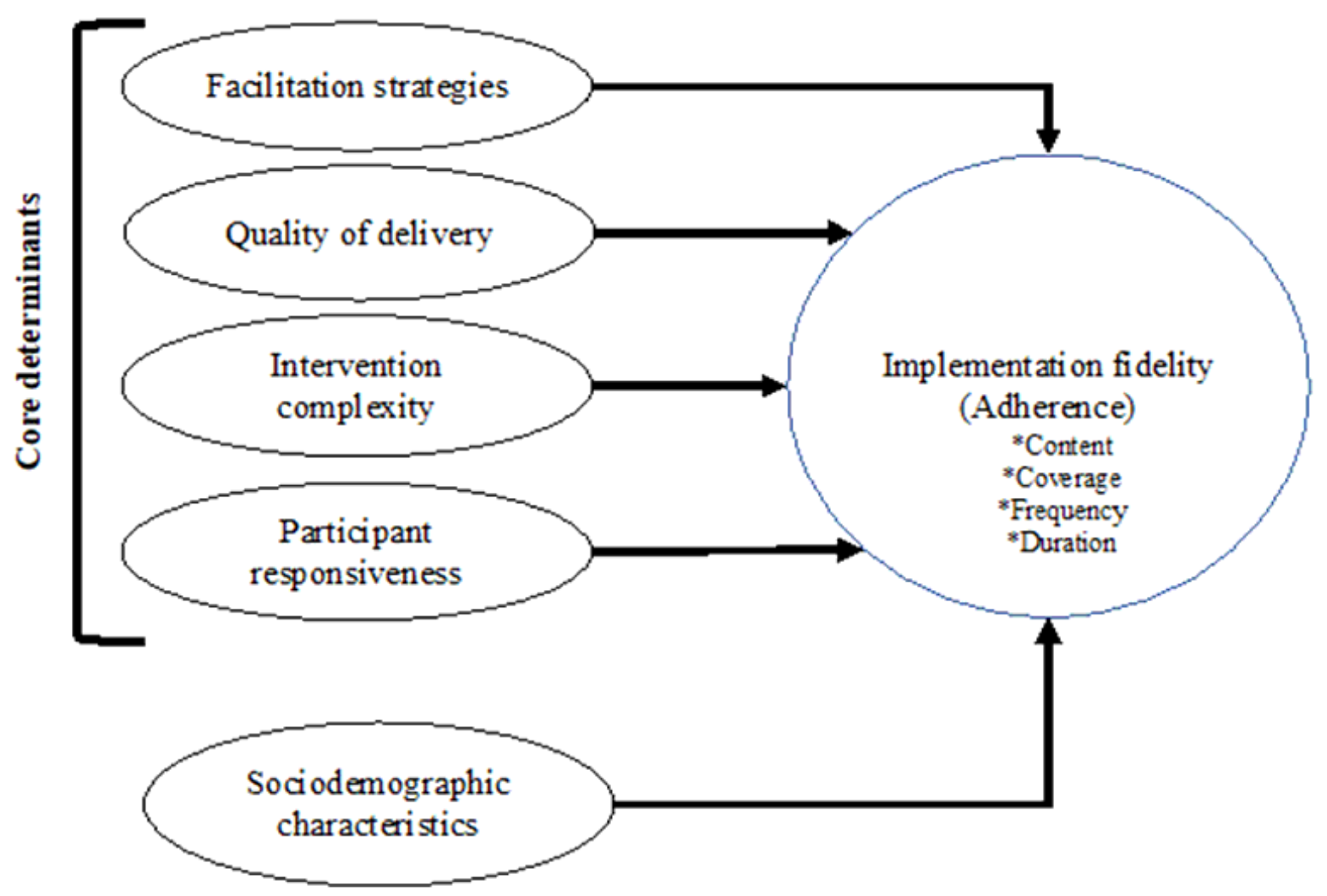

Adapted from Carroll et al, 2007

\section{Figure 1}

The conceptual framework in Figure 1 adapted from Carroll et al. guided the assessment of implementation fidelity and its determinants in this study(Carroll et al., 2007). Implementation fidelity has four constructs of duration, frequency, coverage and content(Ibrahim Muhammad, 2019). The four determinants affecting implementation fidelity according to the framework are: intervention complexity, facilitation strategies, quality of delivery and participant responsiveness(Barat et al., 2001).

\section{Supplementary Files}

This is a list of supplementary files associated with this preprint. Click to download.

- STROBEchecklistv4combined.doc 